\title{
Analysis of Power System Oscillation Frequency Using Differential Groebner Basis and the Harmonic Balance Method
}

\author{
Nan Duan, Bin Wang, Kai Sun \\ Electrical Engineering and Computer Science Department \\ University of Tennessee \\ Knoxville, USA \\ nduan@vols.utk.edu, bwang13@vols.utk.edu, \\ kaisun@utk.edu
}

\author{
Jiaxin Ning \\ Dominion Virginia Power \\ Richmond, VA USA \\ Jiaxin.ning@dom.com
}

\begin{abstract}
For analysis of non-constant electro-mechanical oscillation frequency with a power system, this paper proposes an analytical approach integrating Differential Groebner Basis (Diff-GB) and the Harmonic Balance Method (HBM). This approach is illustrated in detail on a single-machine-infinite-bus system, whose oscillation frequency fluctuates with time due to its nonlinear-system nature. The Diff-GB is first applied to convert the original swing equation into a higher order polynomial differential equation that eliminates explicit sine nonlinearity from its form. Then, the HBM is utilized to derive an approximate analytical expression about oscillation frequency, whose fluctuating nature is then studied by considering multiple oscillation components. The proposed approach is compared with other approaches including small signal analysis, a Taylor Expansion based approach and a numerical approach in terms of oscillation frequency estimation, and demonstrates its superiority.
\end{abstract}

Index Terms-Oscillation frequency, Groebner Basis, Harmonic Balance Method

\section{INTRODUCTION}

The electromechanical oscillation of a power system is tightly related to its angular stability. Following a disturbance, it is crucial to know the oscillation trajectories of all generators' rotor angles and modal features with oscillation, e.g. frequency and damping, in order to judge the angular stability of the system. Currently, the dominating approaches for solving the post-disturbance trajectories of rotor angles in time domain are numerical integration methods, e.g. the Runge-Kutta (R-K) method and the Trapezoidal method. However, a numerical approach may either encounter numerical instability issues (e.g. the R-K method) or be time consuming for a large-scale system. Also, the result is just time-series values of rotor angles at integration steps, and does not provide direct modal information on oscillation. Additional modal analysis techniques are needed to estimate modal features using data over a time window.
Compared to numerical approaches, analytical approaches aim at giving closed-form expressions of rotor angles and other state variables. However, the major obstacle of analytically solving swing equations of a power system is the nonlinearity involving sine functions. Unlike a harmonic oscillator system, oscillation in a power system has nonconstant modal features due to its nonlinear nature [1]. Ref. [2] shows that oscillation frequencies decrease when the system state approaches to the boundary of angular stability. Thus, in theory, there is no accurate, analytic, closed-form solution of generators' rotor angles by existing approaches. Some linearization-based or Taylor Expansion (TE)-based analytic approaches like small-signal analysis (SSA) study linearized power system models to estimate modal features, which are accurate only at the operating point where the system is linearized. Some recent efforts have applied the Adomian Decomposition Method to derive semi-analytical solutions of a power system as an approximate closed-form function of time and other parameters, which still keep certain nonlinearities [3][4] and hence are more accurate than linearization-based methods.

This paper utilizes the Harmonic Balance Method (HBM) to assume an approximate, analytic expression of oscillation frequencies in order to explore their non-constant nature. The method assumes that the solution of the swing equation has the form of an infinite summation of sine or cosine terms about different frequency components in order to represent the non-constant characteristics of oscillation frequency. By only taking finite frequency components into consideration and substituting the assumed form into the formula from linearized swing equations, the magnitude and frequency of each oscillation component are solved.

There are two factors causing losses of solution accuracy in such an approach, i.e. linearization and truncating of infinite summation. Due to the nonexistence of a closed-form analytic solution, the second loss is inevitable. However, the first loss is merely for making the substitution of the assumed solution feasible because the true composition of sine terms cannot be 
analyzed. The using of Differential Groebner Basis (Diff-GB) helps avoid the first unnecessary loss of accuracy. It mathematically converts the system's nonlinear swing equation into a new higher-order differential equation that does not explicitly contain a sine function but, instead, the multiplication of derivatives of rotor angles. This conversion just changes the way that the system is expressed but keeps the integrity of its nonlinear characteristics. This advantage of Diff-GB enhances the HBM by easily substituting the assumed solution into that new equation, which can be solved without sacrificing any nonlinearity in the same way as the aforementioned linearized equation.

Thus, this paper proposes an HBM and Diff-GB based approach for analysis of oscillation frequency. The effectiveness of the proposed approach is illustrated on the single-machine-infinite-bus (SMIB) system with the classical generator model. In the rest of the paper, sections II and III introduce the concepts of Diff-GB and HBM, respectively, section IV compares the result of the propose approach, the linearization based approach and the numerical approach, and conclusions and future work are given in section $\mathrm{V}$.

\section{DIFFERENTIAL GROEBNER BASIS}

\section{A. Algorithm to derive Diff-GB}

Consider a set of equations. Its solutions are also the solutions to the equations that are obtained by differential, addition and multiplication operations based on this set [5]. Further in terms of solutions, a set of differential equations is equivalent to any other set of equations that generates the same ideal. The simplest one of those equivalent sets of differential equations is called Differential Groebner Basis. The word "simplest" describe the property of the basis that every element of the ideal is reduced to zero with respect to that basis. Such a basis usually contains an equation with one variable due to some ordering of the variables. Such a property of Diff-GB usually gives it the advantage of being easier to solve from a "bottom up" way (i.e. solving equations in the Diff-GB from the one which only contains one variable) than the original highly coupled equations.

The algorithm to calculate a Diff-GB for nonlinear differential polynomials is called StandardBasis [7] as shown in Figure 1.

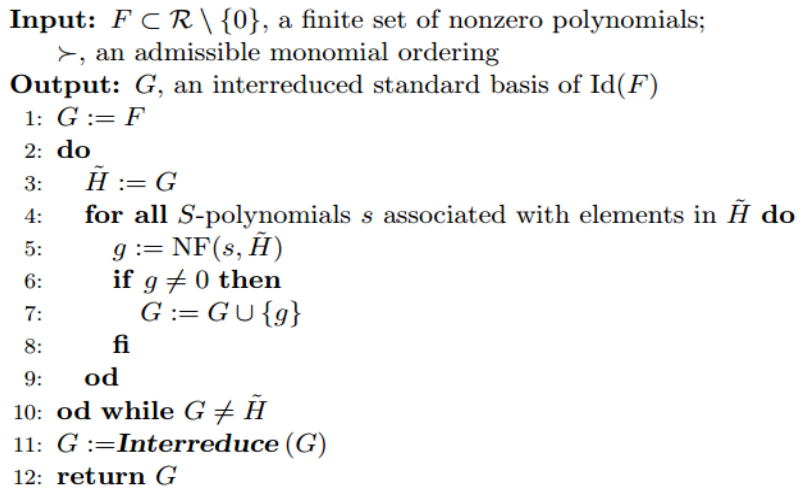

This algorithm is originally designed for difference equations, so the do-while-loop may be infinite in some differential equation cases. Modification such as pseudoreduction [5] is introduced to guarantee termination of the algorithm. Standard Basis has already been well implemented in Maple Differential Algebra package as function called RosenfeldGroebner. In this paper, the calculation of Diff-GB is the result of utilizing the functions with Maple package.

Some systems may contain transcendental functions such as sine and cosine functions. Since their defining differential equations are polynomial, those systems can be reformulated into differential equation system only contain multiplication and addition of the derivatives of those transcendental functions which are treated as new unknowns in the new system. [5]

\section{B. Deriving Diff-GB for the swing equation}

The swing equation of an SMIB system is

$$
M \ddot{\delta}(t)=P_{m}-P_{\max } \sin (\delta(t))
$$

where $M$ is 2 times of the generator inertia divided by synchronous speed $\omega_{R}$ (i.e. $\left.M=2 H / \omega_{R}\right), P_{m}$ is the mechanical power input representing the operation condition and $P_{\max }$ is the maximum steady-state power output of the generator, which is a function of the voltages of the generator EMF and the infinite bus voltage and the impedance in between. Use $f_{1}$ and $f_{2}$ to represent sine and functions, respectively. From trigonometric identities, the swing equation is equivalent to

$$
\left\{\begin{array}{l}
M \ddot{\delta}(t)=P_{m}-P_{\max } f_{1}(t) \\
\dot{f}_{1}(t)-f_{2}(t) \dot{\delta}(t)=0 \\
\dot{f}_{2}(t)+f_{1}(t) \dot{\delta}(t)=0 \\
f_{1}^{2}(t)+f_{2}^{2}(t)=1
\end{array}\right.
$$

Use RosenfeldGroebner function in Maple to calculate its Diff-GB:

$$
\left\{\begin{array}{l}
f_{1}(t)+\frac{M \ddot{\delta}(t)-P_{m}}{P_{\max }}=0 \\
f_{2}(t)+\frac{M \dddot{\delta}(t)}{P_{\max } \dot{\delta}(t)}=0 \\
\dddot{\delta}(t)^{2}+\ddot{\delta}(t)^{2} \dot{\delta}(t)^{2}-\frac{2 P_{m}}{M} \ddot{\delta}(t) \dot{\delta}(t)^{2}+\frac{\left(P_{m}^{2}-P_{\max }^{2}\right)}{M^{2}} \dot{\delta}(t)^{2}=0
\end{array}\right.
$$

Notice that the bottom equation of (3) only contains $\delta$. Moreover, it does not contain any sine or cosine function. This provides the convenience of plugging in any assumption of the solution. However, it is also noticed that the highest order of derivatives becomes 3 , higher than the original 2 . The fact that (1) and (3) having the same solution allows the swing equation to be studied in a new way. 


\section{HARMONIC BALANCE METHOD}

\section{A. Assumption of Solution}

The idea of the Harmonic Balance Method (HBM) is to express the periodic solution of (1) in the form

$$
(t)=A_{n=0}^{N} A_{n} \cos (n t+n)
$$

Then substitute (4) into the bottom equation of (3) and equate the coefficient of each of the lowest $N+1$ harmonics with zero. Thus, $N+1$ algebraic equations about frequency components and amplitudes, $n \omega$ and $A_{n}(n=0 \sim N)$, are obtained.

\section{B. TE and HBM Approach}

To demonstrate the traditional way of using the HBM, apply $3^{\text {rd }}$ order Taylor Expansion (TE) to the right side of (1)

$$
\begin{aligned}
& M \ddot{\delta}(t)=P_{m}-P_{\max }\left(\sin \left(\delta_{0}\right)+\cos \left(\delta_{0}\right)\left(\delta(t)-\delta_{0}\right)\right. \\
& \left.-\sin \left(\delta_{0}\right) \frac{\left(\delta(t)-\delta_{0}\right)^{2}}{2 !}-\cos \left(\delta_{0}\right) \frac{\left(\delta(t)-\delta_{0}\right)^{3}}{3 !}\right)
\end{aligned}
$$

Because this paper only concerns the magnitudes and frequencies of oscillation components, rather than the exact trajectory of solution, as shown in Figure 2, shifting the solution to a standard cosine function can avoid calculation of $A_{0}$ and $\beta$.

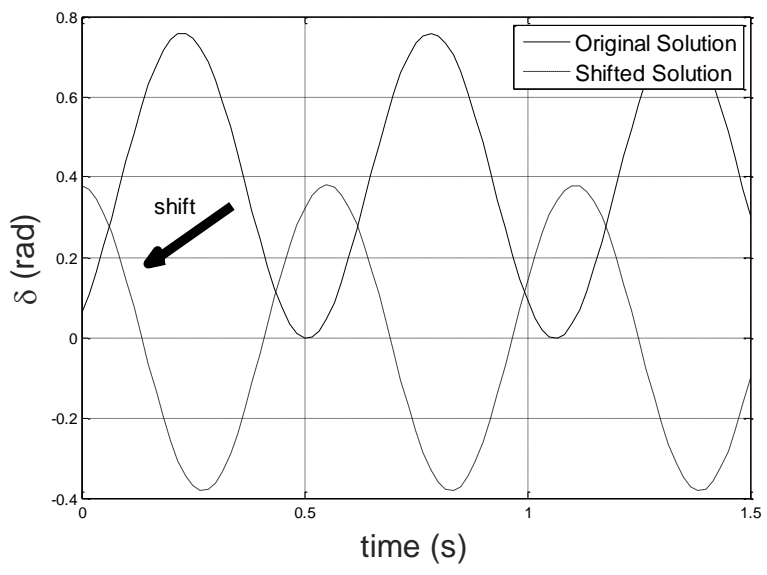

Figure 2. Demonstration of the shifting of numerical solution

Therefore in (4), $n$ starts from 1 and $\beta$ becomes 0 . Keep the first 2 terms of the solution

$$
(t)=A_{1} \cos (t)+A_{2} \cos (2 t)
$$

Substitute (6) into (5) and rewrite it into the form of (7). Its last two terms are a constant term and a sum of higher-order harmonic terms. $C=0$ after the solution is shifted to a standard cosine function. Because by assumption of HBM, $A_{1}>>A_{2}>A_{3} \ldots>A_{N}$, some terms are negligible. Then, considering the initial value, a set of equations is obtained as (8) and is solved for oscillation frequencies.

$$
\begin{aligned}
& \left(P_{\max } A_{1} \cos \left(\delta_{0}\right)-A_{1} M \omega^{2}-\frac{1}{8} P_{\max } A_{1}^{3} \cos \left(\delta_{0}\right)\right. \\
& -\frac{1}{4} P_{\max } A_{1} A_{2}^{2} \cos \left(\delta_{0}\right)+\frac{1}{2} P_{\text {max }} A_{1} A_{2} \delta_{0} \cos \left(\delta_{0}\right) \\
& -\frac{1}{2} P_{\max } A_{1} A_{2} \sin \left(\delta_{0}\right)+P_{\max } A_{1} \delta_{0} \sin \left(\delta_{0}\right) \\
& \left.-\frac{1}{2} P_{\max } A_{1} \delta_{0}^{2} \cos \left(\delta_{0}\right)\right) \cos (\omega t) \\
& +\left(P_{\max } A_{2} \cos \left(\delta_{0}\right)-4 A_{2} M \omega^{2}-\frac{1}{8} P_{\max } A_{2}^{3} \cos \left(\delta_{0}\right)\right. \\
& -\frac{1}{4} P_{\text {max }} A_{1}^{2} A_{2} \cos \left(\delta_{0}\right)+P_{\text {max }} A_{2} \delta_{0} \sin \left(\delta_{0}\right) \\
& -\frac{1}{2} P_{\max } A_{2} \delta_{0}^{2} \cos \left(\delta_{0}\right)+\frac{1}{4} P_{\max } A_{1}^{2} \delta_{0} \cos \left(\delta_{0}\right) \\
& \left.-\frac{1}{4} P_{\max } A_{1}^{2} \sin \left(\delta_{0}\right)\right) \cos (2 \omega t) \\
& +C+f(k \omega)=0
\end{aligned}
$$

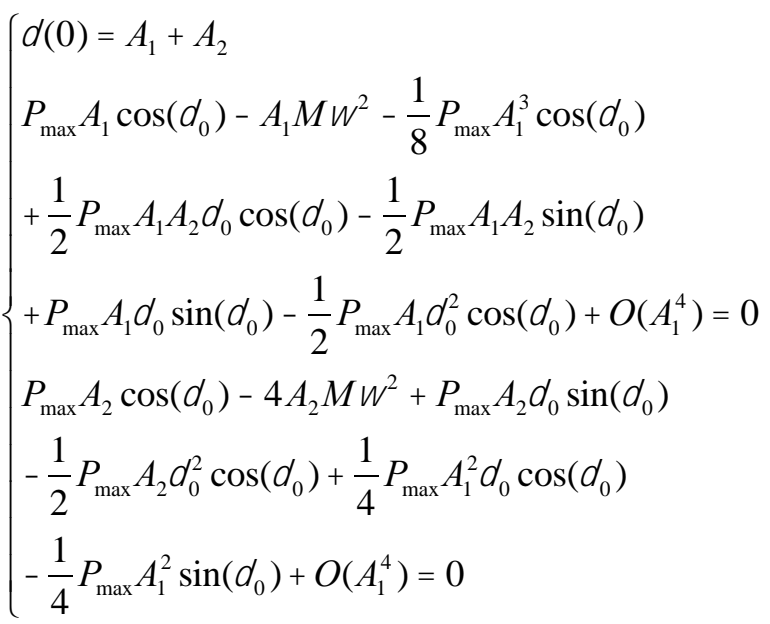

\section{Diff-GB and HBM Approach}

If we assume the solution only has one oscillation frequency, i.e. $N=1$ in (4), there is just one equation

$$
\left(A_{1}^{2}+4\right){ }^{4} M^{2} \quad P_{\max }^{2}+P_{m}^{2}=0
$$

When the solution takes the form of (6), which has two oscillation frequencies, substitute it into the bottom equation of (3) and neglect $O\left(A_{1}^{4}\right)$. Due to the algebraic coupling and the increase in orders of derivatives, there is still only one valid equation as shown in (10).

$$
\frac{1}{2} A_{1}^{2} M P_{m}{ }^{2}+8 A_{2} M^{2}{ }^{4}+2 A_{2} P_{\mathrm{m}}^{2} \quad 2 A_{2} P_{\max }^{2}=0
$$


Following such a procedure, each of the other assumptions about the form of the solution has a corresponding algebraic equation or equation set listed in TABLE I, which can be solved by a numerical approach to estimate average oscillation frequencies over a period of oscillation changing with the operating condition. Note that such an average frequency is not the same frequency calculated from (11) based on the SSA because the oscillation trajectory is ideally sinusoidal.

$$
{ }_{n}=\sqrt{\frac{{ }_{R} P_{\max } \cos { }_{0}}{2 H}}
$$

TABLE I. HBM ALGEBRAIC EQUATIONS

\begin{tabular}{|c|c|}
\hline Solution & Algebraic Equation Set \\
\hline $\begin{array}{c}(t)= \\
A_{1} \cos (t)\end{array}$ & $\begin{array}{l}A_{1} \quad(0)=0 \\
\left(A_{1}^{2}+4\right){ }^{4} M^{2} \quad P_{\max }^{2}+P_{m}^{2}=0\end{array}$ \\
\hline $\begin{array}{l}(t)= \\
A_{1} \cos (t) \\
+A_{2} \cos (2 t)\end{array}$ & $\begin{array}{l}A_{1}+A_{2}-\delta(0)=0 \\
-A_{1} \omega^{2}-4 A_{2} \omega^{2}-\ddot{\delta}(0)=0 \\
\frac{1}{2} A_{1}^{2} M P_{m} \omega^{2}+8 A_{2} M^{2} \omega^{4}+2 A_{2} P_{\mathrm{m}}^{2}-2 A_{2} P_{\max }^{2}=0\end{array}$ \\
\hline $\begin{array}{l}(t)= \\
A_{1} \cos (t) \\
+A_{2} \cos (2 t) \\
+A_{3} \cos (3 t)\end{array}$ & $\begin{array}{l}A_{1}+A_{2}+A_{3}-\delta(0)=0 \\
-A_{1} \omega^{2}-4 A_{2} \omega^{2}-9 A_{3} \omega^{2}-\ddot{\delta}(0)=0 \\
\frac{1}{2} A_{1}^{2} M P_{m} \omega^{2}+8 A_{2} M^{2} \omega^{4}+2 A_{2} P_{\mathrm{m}}^{2}-2 A_{2} P_{\max }^{2}=0 \\
-\frac{1}{2} A_{1} P_{\max }^{2}-3 A_{3} P_{m}^{2}-27 A_{3} M^{2} \omega^{4}+\frac{1}{2} A_{1} P_{m}^{2} \\
+\frac{1}{2} A_{1} M^{2} \omega^{4}+3 A_{3} P_{\max }^{2}=0\end{array}$ \\
\hline $\begin{array}{l}(t)= \\
A_{1} \cos (t) \\
+A_{2} \cos (2 t) \\
+A_{3} \cos (3 t) \\
+A_{4} \cos (4 t)\end{array}$ & $\begin{array}{l}A_{1}+A_{2}+A_{3}+A_{4}-\delta(0)=0 \\
-A_{1} \omega^{2}-4 A_{2} \omega^{2}-9 A_{3} \omega^{2}-16 A_{4} \omega^{2}-\ddot{\delta}(0)=0 \\
\frac{1}{2} A_{1}^{2} M P_{m} \omega^{2}+8 A_{2} M^{2} \omega^{4}+2 A_{2} P_{\mathrm{m}}^{2}-2 A_{2} P_{\max }^{2}=0 \\
-\frac{1}{2} A_{1} P_{\max }^{2}-3 A_{3} P_{m}^{2}-27 A_{3} M^{2} \omega^{4}+\frac{1}{2} A_{1} P_{m}^{2} \\
+\frac{1}{2} A_{1} M^{2} \omega^{4}+3 A_{3} P_{\max }^{2}=0 \\
-8 A_{2} M^{2} \omega^{4}+64 A_{4} M^{2} \omega^{4}-2 A_{2} P_{m}^{2}+2 A_{2} P_{\max }^{2} \\
+4 A_{4} P_{m}^{2}-4 A_{4} P_{\max }^{2}-\frac{1}{2} A_{1}^{2} M P_{m} \omega^{2}=0\end{array}$ \\
\hline
\end{tabular}

If the solution trajectory is not shifted to a standard cosine function, the solution has to be assumed as (4) and there are two more variables, i.e. $A_{0}$ and $\beta$, to solve. This leads to the consideration of two more initial conditions $\dot{\delta}(0)$ and $\dddot{\delta}(0)$. Such high order derivatives require faster sampling frequency $f_{s}$ in numerical integration to acquire accurate enough initial conditions. What specific numerical integration technique to use for solution of each equation set is out of scope of this paper, so all the results are based on the shift of the solution trajectory according to standard cosine function.

\section{RESULTS COMPARISON}

The results of comparing Diff-BG\&HBM approach with the TE\&HBM approach and R-K4 approach are listed in TABLE II. In R-K4 simulation, a $0.05 \mathrm{~s}$ duration line fault is applied. In the TE\&HBM approach, Taylor polynomials are kept up to the $3^{\text {rd }}$ order as in (5). In both of the TE\&HBM and Diff-GB\&HBM approaches, $O\left(A_{1}^{4}\right)$ terms are omitted in algebraic equations. In the R-K4 approach, the oscillation frequencies are defined as the reciprocal of the period between the first two peaks as shown in Figure. 3.

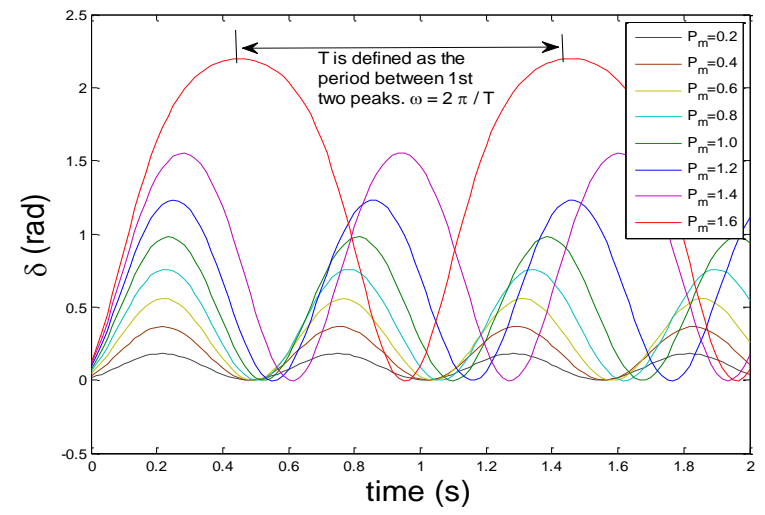

Figure 3. Oscillatory rotor angles under different operation conditions

The estimated oscillation frequencies by the DiffGB\&HBM and TE\&HBM approaches under different operating conditions are listed and compared in TABLE II.

TABLE II. OSCILLATION FREQUENCIES ESTIMATION COMPARISON

\begin{tabular}{|c|c|c|c|c|c|c|c|}
\hline \multirow{2}{*}{\multicolumn{2}{|c|}{$\begin{array}{c}\text { Operation } \\
\text { condition } \\
P_{m}(\mathrm{pu})\end{array}$}} & \multicolumn{4}{|c|}{$\begin{array}{l}\text { Estimated Oscillation frequencies } \\
(\mathrm{rad} / \mathrm{s}) \text { regarding to } N\end{array}$} & \multirow[t]{2}{*}{$\begin{array}{l}\text { Error } \\
(\mathrm{rad} / \mathrm{s})\end{array}$} & \multirow[t]{2}{*}{$\begin{array}{l}\text { R-K4 } \\
(\mathrm{rad} / \mathrm{s})\end{array}$} \\
\hline & & $N=1$ & $N=2$ & $N=3$ & $N=4$ & & \\
\hline \multirow[t]{2}{*}{0.2} & $\mathrm{TE}$ & 11.81 & 11.81 & 11.81 & 11.81 & 0.03 & \multirow[t]{2}{*}{11.78} \\
\hline & GB & 11.79 & 11.64 & 11.68 & 11.68 & 0.10 & \\
\hline \multirow[t]{2}{*}{0.4} & $\mathrm{TE}$ & 11.79 & 11.79 & 11.79 & 11.79 & 0.01 & \multirow[t]{2}{*}{11.78} \\
\hline & GB & 11.69 & 11.54 & 11.58 & 11.58 & 0.20 & \\
\hline \multirow[t]{2}{*}{0.6} & TE & 11.76 & 11.76 & 11.76 & 11.76 & 0.34 & \multirow[t]{2}{*}{11.42} \\
\hline & GB & 11.54 & 11.50 & 11.52 & 11.52 & 0.10 & \\
\hline \multirow[t]{2}{*}{0.8} & TE & 11.73 & 11.73 & 11.73 & 11.73 & 0.31 & \multirow[t]{2}{*}{11.42} \\
\hline & GB & 11.31 & 11.05 & 11.14 & 11.14 & 0.28 & \\
\hline \multirow[t]{2}{*}{1.0} & TE & 11.69 & 11.69 & 11.69 & 11.69 & 0.92 & \multirow[t]{2}{*}{10.77} \\
\hline & GB & 11.00 & 10.77 & 10.94 & 10.94 & 0.17 & \\
\hline \multirow[t]{2}{*}{1.2} & TE & 11.65 & 11.65 & 11.65 & 11.65 & 1.18 & \multirow[t]{2}{*}{10.47} \\
\hline & GB & 10.60 & 10.25 & 10.40 & 10.40 & 0.07 & \\
\hline \multirow[t]{2}{*}{1.4} & TE & 11.62 & 11.62 & 11.62 & 11.62 & 2.20 & \multirow[t]{2}{*}{9.42} \\
\hline & GB & 10.05 & 9.47 & 9.71 & 9.71 & 0.29 & \\
\hline \multirow[t]{2}{*}{1.6} & TE & Null & Null & Null & Null & Null & \multirow[t]{2}{*}{6.08} \\
\hline & GB & Null & Null & 6.38 & 6.38 & 0.30 & \\
\hline
\end{tabular}

Those Nulls with $P_{m}=1.6$ imply there is no reasonable root existing with $N=1$ and 2 cases. In general, based on the 
assumption of the HBM, the rules of searching a reasonable root are listed below:

- Only keep real-value roots and ignore complex roots.

- Only keep roots that satisfy $A_{1}>>A_{2}>A_{3}>\ldots>A_{N}$

- If the $A_{1}$ with a frequency component is larger than 1 , then that frequency is not reasonable, because $A_{1}>1$ means $N$ oscillation components are not enough to decompose the solution. In the assumed form of solution, at least $N+1$ components are needed to decompose the base frequency component's magnitude so as to make $A_{1}$ smaller and eventually less than 1 .

- When there are multiple $A_{1}$ 's $<1$, select the one closest to the initial value of the shifted solution. (i.e. the actual oscillation magnitude)

Figure 4 shows how the oscillation frequencies are estimated by three approaches with the variation of the operating conditions, i.e. $P_{m}$. The results based on the SSA in (11) are also given in Figure 4 to compare with those of the three approaches.

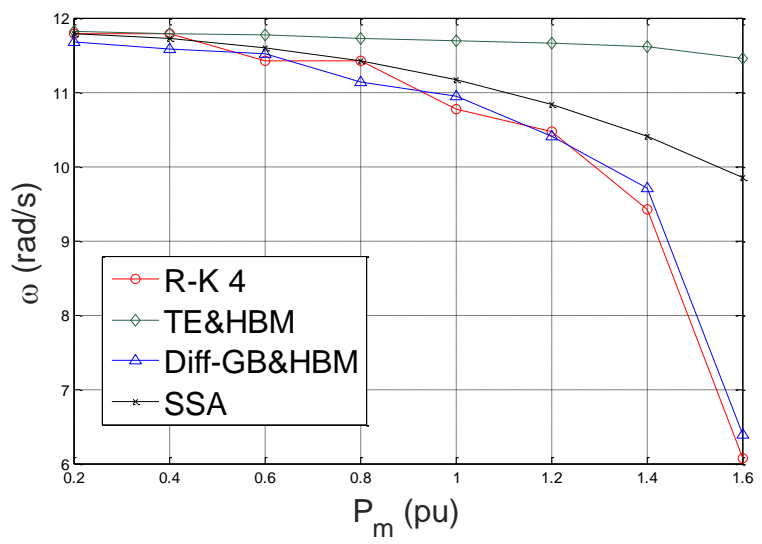

Figure 4. Oscillation frequencies comparison between different estimation approaches

As shown in Figure 4, the TE\&HBM approach is only able to change linearly regarding the change of the operation conditions. While, compared with the TE\&HBM and the SSA approaches, the Diff-GB\&HBM approach more accurately depicts the nonlinear variation of oscillation frequencies and matches very well with the numerical approach, which is regarded as the benchmark. Moreover, the fact that the DiffGB\&HBM result is superior over the TE\&HBM and the SSA results indicates that the Diff-GB can fully exploit the HBM's ability of analytically estimating oscillation frequencies of a nonlinear power system.

The Diff-GB\&HBM approach preserves the nonlinearity of the system, because its system representation is more accurate. Therefore, the estimation of frequency is more accurate. If the same goal needs to be achieved in TE\&HBM approach, higher order Taylor polynomials and HBM coefficient terms need to be adopted. However, this effort will lead to a deterioration of computational efficiency. The computational advantage of neglecting more high order terms is that, while solving the simplified algebraic equation set, fewer roots need to be solved such that tremendous computational time can be saved. After those roots are solved, the space of searching for reasonable roots would also be smaller. Thus, the efficiency of searching a reasonable solution is largely enhanced. However, when TE is used, even if higher order Taylor polynomials and HBM coefficients terms are kept in HBM (considering up to $5^{\text {th }}$ order Taylor polynomials and neglecting only $O\left(A_{1}{ }^{6}\right)$ terms in HBM coefficients) and sacrifice of the computational efficiency is accepted (the increase of time for finding all roots almost grow exponentially), the improvement of accuracy would be very marginal.

A problem with the Diff-GB is that it increases the highest order of derivatives in the swing equation. So, while solving those parameters in an assumed solution, it needs initial values of higher order angle derivatives, which may need to be acquired from numerical integration. If data sampling frequency $f_{s}$ is not fast enough, the calculation of higher order derivatives in numerical integration would be inaccurate. Future work is desirable to estimate more reliable and accurate initial values.

\section{CONCLUSION}

This paper proposes a new analytical Diff-GB\&HBM approach to estimate power system oscillation frequency. This approach takes the advantage of the Diff-GB to preserve the nonlinearity of the swing equation, and, thus, provides the freedom to use HBM to an intact system model. The new approach is compared to a TE-based approach and a numerical approach. The Diff-GB\&HBM approach can match well with the result of the numerical approach, which is considered as the benchmark. The merit of the Diff-GB\&HBM approach is to analytically represent the non-constant characteristics of an oscillation. The future work will validate this new approach to multi-machine power systems.

\section{REFERENCES}

[1] A. Nayfeh, D. Mook, Nonlinear Oscillations, New York: Wiley, 1979, p. 59-63.

[2] B. Wang, K. Sun, et al. "A study on fluctuations in electromechanical oscillation frequencies of power systems." 2014 IEEE PES General Meeting, National Harbor, MD

[3] N. Duan, K. Sun, "Application of the Adomian Decomposition Method for Semi-Analytic Solutions of Power System Differential Algebraic Equations", PowerTech 2015

[4] N. Duan, K. Sun, "Finding Semi-Analytic Solutions of Power System Differential-Algebraic Equations for Fast Transient Stability Simulation", IEEE Trans. Power Systems, under review

[5] E. Mansfield, "Differential Groebner Bases", Ph.D. dissertation, Univ. of Sydney, Sydney, Australia, 1991.

[6] J. Liu, J. Peddieson, "Application of Groebner Basis Methodology to Nonlinear Mechanics Problems." Mathematical Software-ICMS 2014. Springer Berlin Heidelberg, 2014. 398-405.

[7] V. Gerdt, D. Robertz, "Computation of Difference Groebner Bases", Computer Science Journal of Moldova, vol.20, no.2(59), 2012.

[8] P.M. Anderson and A.A. Fouad, Power system control and stability. 2nd ed., New York: Wiley Interscience, 2003, p.13-66. 